\title{
COMPLEX SYMMETRY OF TOEPLITZ OPERATORS
}

\author{
YONG CHEN, YOUNG JOO LEE, AND YILE ZHAO
}

\begin{abstract}
We introduce a new class of conjugations and characterize complex symmetric Toeplitz operators on the Hardy space with respect to those conjugations. Also, we prove that complex symmetricity and UET property are the same for a certain class of Toeplitz operators. We also discuss the analytic symmetricity for Toeplitz operators. Our results extend several known results by providing unified ways of treating them.
\end{abstract}

\section{INTRODUCTION}

Given a complex separable Hilbert space $\mathcal{H}$ endowed with the inner product $\langle,\rangle_{\mathcal{H}}$, a conjugate-linear map $C: \mathcal{H} \rightarrow \mathcal{H}$ is called a conjugation if $C^{2}=I$ and $\langle C x, C y\rangle_{\mathcal{H}}=\langle y, x\rangle_{\mathcal{H}}$ for all $x, y \in \mathcal{H}$. Given a bounded linear operator $T$ and conjugation $C$ on $\mathcal{H}, T$ is called $C$ symmetric if $C T C=T^{*}$. Also, we say that $T$ is complex symmetric if $T$ is $C$-symmetric for some conjugation $C$ on $\mathcal{H}$. The term "complex symmetric" is due to the fact that $T$ is complex symmetric if and only if $T$ is unitarily equivalent to a symmetric matrix with complex entries regarded as an operator acting on an $\ell^{2}$-space of the appropriate dimension.

The class of complex symmetric operators is very large and includes not only normal operators but also lots of nonnormal operators containing Hankel operators, truncated Toeplitz operators and Volterra integration operators. In order to obtain a better understanding for complex symmetric operators, a lot of effort are devoted to the study of complex symmetricity for several kinds of operators containing partial isometries [7], weighted shifts [20], triangular operators [19] and composition operators [11, 12, 15, 16, 18].

Recently, it has been studied the problem of when a Toeplitz operator on the Hardy space is complex symmetric. We first recall the Hardy

Date: July 15, 2021.

2010 Mathematics Subject Classification. Primary 47B35; Secondary 46E22, 47B99.

Key words and phrases. Toeplitz operator, Hardy space, complex symmetry, canonical symmetry, analytic symmetry. 
space. Let $\mathbb{T}$ be the boundary of the open unit disk $\mathbb{D}$ in the complex plane $\mathbb{C}$. We let $L^{2}=L^{2}(\mathbb{T}, \sigma)$ be the usual Lebesgue space on $\mathbb{T}$ where $\sigma$ is the normalized Haar measure on $\mathbb{T}$. The classical Hardy space $H^{2}$ is consisting of all analytic functions $f$ on $\mathbb{D}$ satisfying

$$
\sup _{0 \leq r<1} \int_{\mathbb{T}}|f(r \xi)|^{2} d \sigma(\xi)<\infty .
$$

As is well known, the Hardy space $H^{2}$ is isometrically identified with a closed subspace of $L^{2}$ via the boundary functions. Thus, we will use the same letter for a function in $H^{2}$ and its boundary function in $L^{2}$. In particular, $H^{2}$ is a Hilbert space with the inner product

$$
\langle f, g\rangle=\int_{\mathbb{T}} f \bar{g} d \sigma
$$

for functions $f, g \in H^{2}$. Also, it is well known that the Hilbert space orthogonal projection $P$ from $L^{2}$ onto $H^{2}$ is realized by the integral operator

$$
P \psi(z)=\left\langle\psi, K_{z}\right\rangle=\int_{\mathbb{T}} \frac{\psi(\xi)}{1-z \bar{\xi}} d \sigma(\xi), \quad z \in \mathbb{D}
$$

for $\psi \in L^{2}$ where $K_{z}$ is the reproducing kernel on $H^{2}$ given by

$$
K_{z}(\xi)=\frac{1}{1-\bar{z} \xi}, \quad \xi \in \mathbb{T} .
$$

Given $\phi \in L^{\infty}(\mathbb{T})$, the Toeplitz operator $T_{\phi}$ with symbol $\phi$ is defined by

$$
T_{\phi} f=P(\phi f)
$$

for $f \in H^{2}$. Clearly the Toeplitz operator $T_{\phi}$ is a bounded linear operator on $H^{2}$.

As is well known, for $\phi \in L^{\infty}(\mathbb{T}), T_{\phi}$ is normal on $H^{2}$ if and only if $\phi=f+\overline{\gamma f}$ for some $f \in H^{\infty}$ and $\gamma \in \mathbb{T}$; see [1] for details. So, such $\phi$ induces a complex symmetric Toeplitz operator. Guo and Zhu 9] characterized a certain trigonometric symbol for which the corresponding Toeplitz operator is complex symmetric. They also raised an interesting question at the same paper: Characterize complex symmetric Toeplitz operators on $H^{2}$. Generally it seems to be difficult to describe when a Toeplitz operator is complex symmetric. Recently several kinds of conjugations have been considered and then the characterizing problem of complex symmetric Toeplitz operators with respect to those conjugations has been studied.

Recently, Ko and Lee [13] considered a class of conjugations and studied complex symmetric Toeplitz operators. More explicitly, given 
$\lambda \in \mathbb{T}$, define

$$
C_{\lambda} h(z)=\overline{h(\lambda \bar{z})}, \quad z \in \mathbb{T}
$$

for functions $h \in L^{2}$. In other words, if we write $h=\sum_{j=-\infty}^{\infty} c_{n} z^{n}$ for the Fourier series expansion of $h, C_{\lambda} h$ can written as

$$
C_{\lambda} h=\sum_{n=-\infty}^{\infty} \overline{c_{n}} \overline{\lambda^{n}} z^{n} .
$$

Then, one can easily see that $C_{\lambda}$ is a conjugation on both $L^{2}$ and $H^{2}$. Given $\phi \in L^{\infty}(\mathbb{T})$ and $\lambda \in \mathbb{T}$, Ko and Lee proved that the Toeplitz

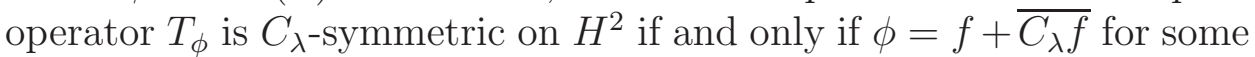
$f \in H^{2}$, which is also equivalent to $\phi(\lambda z)=\phi(\bar{z})$ for all $z \in \mathbb{D}$; see [13] for details.

Recently, a more general class of conjugations induced by sequences has been introduced in [14. Given a sequence $\alpha=\left(\alpha_{0}, \alpha_{1}, \alpha_{2}, \cdots\right)$ where $\alpha_{n} \in \mathbb{T}$, define a conjugation $C_{\alpha}$ by

$$
C_{\alpha} h=\sum_{n=0}^{\infty} \overline{c_{n}} \alpha_{n} z^{n}
$$

for $h=\sum_{n=0}^{\infty} c_{n} z^{n} \in H^{2}$. Specially, $C_{\alpha}=C_{\lambda}$ when $\alpha_{n}=\overline{\lambda^{n}}$ for each $n$. Such a conjugation is known to be a canonical one. Recall that a conjugation $C$ is called a canonical conjugation if $C z^{n} \in \vee\left\{z^{n}\right\}$ for all $n=0,1,2, \cdots$ where $\vee$ denotes the closed linear span. Given a conjugation $C$ on $H^{2}$, we note that $C$ is a canonical conjugation if and only if $C=C_{\alpha}$ for some $\alpha=\left\{\alpha_{n}\right\}$ where $\alpha_{n} \in \mathbb{T}$. An operator is called canonically symmetric if it is complex symmetric with respect to a canonical conjugation.

In a recent paper [14, the authors characterized canonically symmetric Toeplitz operators in terms of the notion of geometric pair sequences; see Theorem 2.11 of [14] for details. Very recently, it turned out that canonically symmetricity and $C_{\lambda}$-symmetricity are the same for Toeplitz operators. More explicitly, given $\phi \in L^{\infty}(\mathbb{T})$, it is proved that $T_{\phi}$ is canonically symmetric if and only if $T_{\phi}$ is $C_{\lambda}$-symmetric for some $\lambda \in \mathbb{T}$, which provides a finer version of the result in [14]; see [2] for details and related results.

Motivated by these results, we introduce a more general class of conjugations induced by a sequence and an inner function. To be more precise, we first need some notations. Let $\mathscr{M}$ be the set of all sequences $\alpha=\left(\alpha_{0}, \alpha_{1}, \alpha_{2}, \cdots\right)$ where each $\alpha_{n} \in \mathbb{T}$. Also, the notation $H^{\infty}$ denotes the space of all bounded analytic functions on $\mathbb{D}$ and a function $u \in H^{\infty}$ is said to be inner if $|u|=1$ a.e. on $\mathbb{T}$. 
Let $\alpha=\left\{\alpha_{m}\right\} \in \mathscr{M}$ and $u$ be a nonconstant inner function. Choose an orthonormal basis (briefly, ONB) $E=\left\{e_{j}: j \in \Lambda\right\}$ of the model space $H^{2} \ominus u H^{2}$. By the Wold decomposition theorem, we have

$$
H^{2}=\vee\left\{u^{m} e_{j}: j \in \Lambda, m=0,1,2, \cdots\right\} .
$$

We define $C_{\alpha, u, E}$ on $H^{2}$ by

$$
C_{\alpha, u, E}\left(\sum_{m, j} \mu_{m, j} u^{m} e_{j}\right)=\sum_{m, j} \overline{\mu_{m, j}} \alpha_{m} u^{m} e_{j} .
$$

Then, one can check that $C_{\alpha, u, E}$ is a conjugation on $H^{2}$. Note that the

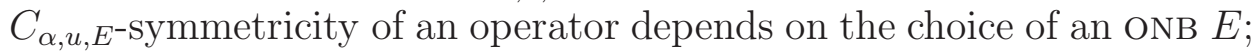
see Example 2.4. We say that an operator $T$ on $H^{2}$ is $C_{\alpha, u^{-}}$symmetric

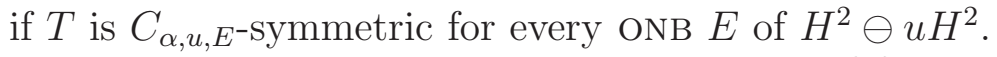

In the special case when $u=z$ and $E=\{1\}$, the corresponding conjugation $C_{\alpha, u, E}$ is a canonical conjugation $C_{\alpha}$. More specially, if $\alpha$ is given by a geometric sequence such as $\alpha_{n}=\bar{\lambda}^{n}$ for $\lambda \in \mathbb{T}$, the corresponding conjugation $C_{\alpha, u, E}$ is just conjugation $C_{\lambda}$. The $C_{\alpha, u}$ will be denoted by $C_{\lambda, u}$ if $\alpha$ is given by a geometric sequence induced by $\lambda \in \mathbb{T}$ above.

For conjugations $C_{\alpha, u, E}$, we first obtain the following characterization of when a Toeplitz operator is $C_{\alpha, u}$-symmetric on $H^{2}$.

Theorem 1.1. Let $\phi \in L^{\infty}(\mathbb{T})$ and $u$ be inner with a zero in $\mathbb{D}$. Then the following statements are equivalent:

(a) $T_{\phi}$ is $C_{\alpha, u}$-symmetric for some $\alpha \in \mathscr{M}$.

(b) $T_{\phi}$ is $C_{\lambda, u}$-symmetric for some $\lambda \in \mathbb{T}$.

(c) $\phi=f \circ u+\overline{C_{\lambda}(f) \circ u}$ for some $\lambda \in \mathbb{T}$ and $f \in H^{2}$.

We were not able to prove the same in the case when the inner function $u$ has no zero. The proof of Theorem 1.1 will be given at Section 2. As a special case of when an inner function is given by a single Möbius transform, we extend and recover several known results in [2] and [13].

Recall that a bounded linear operator $T$ on a Hilbert space $\mathcal{H}$ is called UET if $T$ is unitary equivalent to $C T^{*} C$ for some conjugation $C$ on $\mathcal{H}$. The notion of UET was inspired by Halmos [10] and has been studied extensively in [8] and [9]. In particular, it plays an important role in describing the block structure of complex symmetric operators.

Note that each complex symmetric operator is UET but the converse is not true generally; see [6] or [8] for details. However, it turns out that the converse is also true for a certain class of Toeplitz operators on $H^{2}$. In [2], it was recently shown that, for a Toeplitz operator with 
certain trigonometric symbol, the complex symmetricity and UET property are the same, which is equivalent to that it is either normal or

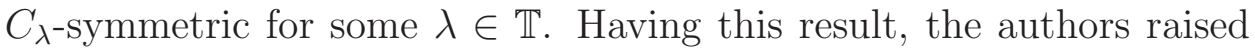
the following questions.

Question 1.2. (a) Is a UET Toeplitz operator complex symmetric?

(b) Is a non-normal complex symmetric Toeplitz operator $C_{\lambda}$-symmetric for some $\lambda \in \mathbb{T}$ ?

For Question 1.2, we consider a more general class of Toeplitz operators with non-trigonometric symbols. For a point $a \in \mathbb{D}$, we let

$$
\varphi_{a}(z)=\frac{a-z}{1-\bar{a} z}, \quad z \in \mathbb{D}
$$

denote the usual Möbius transformation of $\mathbb{D}$. Also, a function of the form $B=\gamma \varphi_{a_{1}} \varphi_{a_{2}} \cdots \varphi_{a_{N}}$ where $\gamma \in \mathbb{T}$ and $a_{j} \in \mathbb{D}$ is called a finite Blaschke product.

Our next result below provides another evidence for a positive answer to (a) of Question 1.2 and a negative answer to (b) of Question 1.2 .

Theorem 1.3. Let $\phi=B_{1}+\overline{\mu B_{2} \circ B_{1}}$ where $\mu \in \mathbb{C}$ and $B_{1}, B_{2}$ are finite Blaschke products. Then the following assertions are equivalent:

(a) $T_{\phi}$ is complex symmetric.

(b) $T_{\phi}$ is UET.

(c) $\mu B_{2}=q \varphi_{b}$ for some $q \in \mathbb{T}$ and $b \in \mathbb{D}$ satisfying $q b=\bar{b}$.

Recently, the complex symmetricity of Toeplitz operators has been studied in terms of a certain geometric property of the symbol. Recall that a closed curve $\gamma$ in $\mathbb{C}$ is called nowhere winding if its winding number at $z$ is 0 for all $z \in \mathbb{C} \backslash \gamma$; see Section 3 for details. Noor [17] recently proved that the image of $\mathbb{T}$ under a continuous symbol of a complex symmetric Toeplitz operator is nowhere winding. Based on this result, Noor posed a question of whether the converse is also true.

Thus, in view of Question 1.2 together with Noor's question above, we notice that there might be some connections between complex symmetricity, UET condition and nowhere winding property. We show that the image $\phi(\mathbb{T})$ of a UET Toeplitz operator with continuous symbol $\phi$ is nowhere winding either; see Proposition 3.2. Also, for symbols of the form $\phi=z+\overline{q \varphi_{b}}$ with $|q| \leq 1$ and $b \in \mathbb{D}$, we show that $T_{\phi}$ is UET if and only if $T_{\phi}$ is complex symmetric, which is also equivalent to that $\phi(\mathbb{T})$ is nowhere winding; see Proposition 3.3. This gives a positive example to Noor's question mentioned above. Those results including Theorem 1.3 will be proved at Section 3 . 
Finally, we have another observation for complex symmetric Toeplitz operators. For $f, g \in H^{\infty}$, put $\phi=f+\bar{g}$. If $T_{\phi}$ is complex symmetric for some conjugation $C$ on $H^{2}$, then

$$
C T_{f} C+C T_{\bar{g}} C=T_{\bar{f}}+T_{g} .
$$

Since $T_{g}, C T_{f} C$ are hyponormal and $C T_{\bar{g}} C, T_{\bar{f}}$ are co-hyponormal, we naturally guess that $C T_{f} C$ is a Toeplitz operator with analytic symbol and $C T_{f} C=T_{g}$. But it is not the case generally; see Example 4.3. So we need a definition.

Given $\phi \in L^{\infty}(\mathbb{T})$, we say that $T_{\phi}$ is analytically symmetric if there exist $f, g \in H^{\infty}$ and a conjugation $C$ on $H^{2}$ such that $C T_{f} C=T_{g}$ and $\phi=f+\bar{g}$. In this case, since $C T_{g} C=T_{f}$ and $C T_{\bar{g}} C=T_{\bar{f}}$, we see

$$
C T_{\phi} C=T_{g+\bar{f}}=T_{\phi}^{*}
$$

Thus, analytic symmetric Toeplitz operators are complex symmetric. But the converse is not true in general; see also Example 4.3. So, we have a question below naturally.

Question 1.4. For which $\phi \in L^{\infty}(\mathbb{T})$, is the Toeplitz operator $T_{\phi}$ analytically symmetric on $H^{2}$ ?

In Section 4, we study Question 1.4 and first show that, for a Toeplitz operator with general trigonometric symbol, the analytic symmetric property is equivalent to the $C_{\lambda^{-}}$symmetry for some $\lambda \in \mathbb{T}$; see Theorem 4.2. Also, we characterize an analytic symmetric Toeplitz operator when the analytic part of the symbol is a polynomial of degree 2; see Theorem 4.4. Finally, in conjunction with the notion of analytic symmetric Toeplitz operator, we characterize conjugations $C$ on $H^{2}$ for which $C T_{f} C$ is a Toeplitz operator with an analytic symbol for any $f \in H^{\infty}$; see Theorem 4.5.

\section{2. $C_{\alpha, u}$-Symmetric Toeplitz operators}

In this section we prove Theorem 1.1 and obtain immediate consequences in case when the inner function is given by a single Möbius transform.

Given $\alpha=\left\{\alpha_{m}\right\} \in \mathscr{M}$ and an ONB $E=\left\{e_{j}: j \in \Lambda\right\}$ of $H^{2} \ominus$ $u H^{2}$ corresponding to a nonconstant inner function $u$, we recall the conjugation $C_{\alpha, u, E}$ on $H^{2}$ defined by

$$
C_{\alpha, u, E}: u^{m} e_{j} \mapsto \alpha_{m} u^{m} e_{j}, \quad j \in \Lambda, m=0,1,2 \cdots .
$$

We also recall that $C_{\alpha, u}$ is denoted by $C_{\lambda, u}$ if $\alpha=\left\{\bar{\lambda}^{m}\right\}$ for some $\lambda \in \mathbb{T}$.

We first observe basic results which will be useful in our characterizations. One of them shows that $T_{\phi}$ is $C_{\lambda}$-symmetric if and only if $T_{\phi \circ u}$ is 
$C_{\lambda, u}$-symmetric, which not only provides a much larger class of complex symmetric Toeplitz operators but also reminds that (b) of Question 1.2 may be true only for Toeplitz operators with trigonometric symbols.

Proposition 2.1. Let $\phi \in L^{\infty}(\mathbb{T})$ and $u$ be a non-constant inner function. Then the following statements are equivalent:

(a) $T_{\phi}$ is canonically symmetric.

(b) $T_{\phi}$ is $C_{\lambda}$-symmetric for some $\lambda \in \mathbb{T}$.

(c) $T_{\phi o u}$ is $C_{\lambda, u}$-symmetric for some $\lambda \in \mathbb{T}$.

(d) $T_{\phi o u}$ is $C_{\lambda, u, E^{-}}$-symmetric for some $\lambda \in \mathbb{T}$ and oNB $E$ of $H^{2} \ominus$ $u H^{2}$.

(e) $\phi=f+\overline{C_{\lambda} f}$ for some $\lambda \in \mathbb{T}$ and $f \in H^{2}$.

(f) $\phi(\lambda z)=\phi(\bar{z})$ for some $\lambda \in \mathbb{T}$.

Proof. First, implications $(\mathrm{a}) \Longleftrightarrow(\mathrm{b}) \Longleftrightarrow(\mathrm{e}) \Longleftrightarrow(\mathrm{f})$ follow from [2] and [13] as mentioned before. Also, (c) $\Longrightarrow(\mathrm{d})$ is obvious. Thus, it remains to prove implications $(\mathrm{e}) \Longrightarrow(\mathrm{c})$ and $(\mathrm{d}) \Longrightarrow(\mathrm{f})$.

First assume (e) and show (c). Fix an ONB $E=\left\{e_{j}: j \in \Lambda\right\}$ of $H^{2} \ominus u H^{2}$. Write $\phi=\sum_{n=-\infty}^{\infty} a_{n} z^{n}$ for the Fourier series expansion of $\phi$. Note $P\left(u^{\ell} e_{j}\right)=0$ for all integer $\ell<0$ and $j \in \Lambda$. It follows that

$$
\begin{aligned}
C_{\lambda, u, E} T_{\phi \circ u} C_{\lambda, u, E}\left(u^{m} e_{j}\right) & =C_{\lambda, u, E} T_{\phi \circ u}\left(\bar{\lambda}^{m} u^{m} e_{j}\right) \\
& =\lambda^{m} C_{\lambda, u, E} P\left(\sum_{n=-\infty}^{\infty} a_{n} u^{n+m} e_{j}\right) \\
& =\lambda^{m} C_{\lambda, u, E}\left(\sum_{n=-m}^{\infty} a_{n} u^{n+m} e_{j}\right) \\
& =\lambda^{m} \sum_{n=-m}^{\infty} \overline{a_{n}} \overline{\lambda^{n+m}} u^{n+m} e_{j} \\
& =P\left(\sum_{n=-\infty}^{\infty} \overline{a_{n}} \overline{\lambda^{n}} u^{n+m} e_{j}\right) \\
& =P\left[\left(\left(C_{\lambda} \phi\right) \circ u\right) u^{m} e_{j}\right] \\
& =T_{\left(C_{\lambda} \phi\right) \circ u}\left(u^{m} e_{j}\right)
\end{aligned}
$$

for all $m, j$ and hence

$$
C_{\lambda, u, E} T_{\phi \circ u} C_{\lambda, u, E}=T_{\left(C_{\lambda} \phi\right) \circ u}
$$


Now, since $\phi=f+\overline{C_{\lambda} f}$ by the assumption, (2.1) shows that

$$
\begin{aligned}
C_{\lambda, u, E} T_{\phi \circ u} C_{\lambda, u, E} & =T_{C_{\lambda}\left(f+\overline{C_{\lambda} f}\right) \circ u} \\
& =T_{C_{\lambda}\left(f+C_{\lambda} \bar{f}\right) \circ u} \\
& =T_{\left(C_{\lambda} f+\bar{f}\right) \circ u} \\
& =T_{\phi \circ u}^{*} .
\end{aligned}
$$

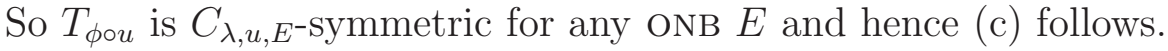

Now assume (d) and show (e). Since $T_{\phi \circ u}$ is $C_{\lambda, u, E^{-S y m m e t r i c, ~ i t ~}}$ follows from (2.1) that $T_{\left(C_{\lambda} \phi\right) \circ u}=T_{\overline{\phi \circ u}}$, which implies $\left(C_{\lambda} \phi\right) \circ u=\overline{\phi \circ u}$. Hence $C_{\lambda} \phi=\bar{\phi}$ and (f) holds. The proof is complete.

Before we prove Theorem 1.1, we need some facts. Fix a point $a \in \mathbb{D}$. Let $e_{a}=\sqrt{1-|a|^{2}} K_{a}$ be the normalized kernel and define an operator $U_{a}$ on $H^{2}$ by $U_{a} f=\left(f \circ \varphi_{a}\right) e_{a}$ for $f \in H^{2}$. Then, using the fact $\left(e_{a} \circ \varphi_{a}\right) e_{a}=1$, one can easily check that $U_{a}$ is a unitary operator and

$$
U_{a}^{*}=U_{a}^{-1}=U_{a}, \quad U_{a} T_{\phi} U_{a}=T_{\phi \circ \varphi_{a}} .
$$

Thus $T_{\phi} \cong T_{\phi \circ \varphi_{a}}$. Here and in what follows, the notation $\cong$ denotes unitary equivalence as usual. Also, for a conjugation $C$ on $H^{2}$, noting $U_{a} C U_{a}$ is another conjugation on $H^{2}$, we see that $T_{\phi}$ is $C$-symmetric if and only if $T_{\phi \circ \varphi_{a}}$ is $U_{a} C U_{a}$-symmetric.

Now we are ready to prove Theorem 1.1 .

Proof of Theorem 1.1. First assume (c) and show (b). By an application of (2.1), we get $C_{\lambda, u, E} T_{\phi} C_{\lambda, u, E}=T_{\phi}^{*}$ for any ONB $E$ of $H^{2} \ominus u H^{2}$, so (b) holds. Also, implication (b) $\Longrightarrow$ (a) follows from taking $\alpha_{m}=\overline{\lambda^{m}}$ for each $m$.

We finally assume (a) and show (c). Since $u$ has a zero in $\mathbb{D}$, let $u(a)=0$ for some $a \in \mathbb{D}$. It is easy to check that $E$ is an ONB of $H^{2} \ominus u H^{2}$ if and only if $U_{a} E$ is an ONB of $H^{2} \ominus\left(u \circ \varphi_{a}\right) H^{2}$. Also, we note that $U_{a} C_{\alpha, u, E} U_{a}=C_{\alpha, u \circ \varphi_{a}, U_{a} E}$. Thus, as mentioned before, $T_{\phi}$ is $C_{\alpha, u}$-symmetric if and only if $T_{\phi \circ \varphi_{a}}$ is $C_{\alpha, u \circ \varphi_{a}}$-symmetric. Since $u \circ \varphi_{a}(0)=0$, the observation above shows that we may assume $a=0$ without loss of generality. Thus, $u(0)=0$ and so $1 \in H^{2} \ominus u H^{2}$. Choose an oNB $E=\left\{1, e_{1}, e_{2}, \cdots\right\}$ of $H^{2} \ominus u H^{2}$. Since $H^{2}=\bigvee_{m \geq 0} u^{m} E$, we may write

$$
\phi=\sum_{j \geq 0}\left(a_{j} u^{j}+b_{j} \bar{u}^{j}\right)+\sum_{k \geq 1} \sum_{j \geq 0}\left(\eta_{k, j} u^{j} e_{k}+\beta_{k, j} \bar{u}^{j} \overline{e_{k}}\right) .
$$

Let $\alpha=\left\{\alpha_{m}\right\}$. Since $T_{\phi}$ is $C_{\alpha, u, E^{-S y m m e t r i c, ~ w e ~ h a v e ~ f o r ~ a n y ~ i n t e g e r s ~}}$ $n, m \geq 0$,

$$
\left\langle C_{\alpha, u, E} T_{\phi} C_{\alpha, u, E} u^{m}, u^{n} e_{\ell}\right\rangle=\left\langle T_{\bar{\phi}} u^{m}, u^{n} e_{\ell}\right\rangle
$$


for all integers $\ell \geq 1$, which implies that for $n \geq m$,

$$
\overline{\alpha_{m}} \alpha_{n} \overline{\eta_{\ell, n-m}}=\overline{\beta_{\ell, n-m}}, \quad \ell \geq 1 \text {. }
$$

It follows that

$$
\beta_{\ell, j}=\alpha_{m} \overline{\alpha_{m+j}} \eta_{\ell, j}
$$

for all $m \geq 0, \ell \geq 1$ and $j \geq 0$.

Fix $\gamma \in \mathbb{T}$ and set $E_{\gamma}=\left\{1, \gamma e_{1}, \gamma e_{2}, \cdots\right\}$. It is clear that $E_{\gamma}$ is also an ONB of $H^{2} \ominus u H^{2}$. We can rewrite $\phi$ with respect to $E_{\gamma}$ as

$$
\phi=\sum_{j \geq 0}\left(a_{j} u^{j}+b_{j} \bar{u}^{j}\right)+\sum_{k \geq 1} \sum_{j \geq 0}\left(\bar{\gamma} \eta_{k, j} u^{j} \gamma e_{k}+\gamma \beta_{k, j} \bar{u}^{j} \overline{\gamma e_{k}}\right) .
$$

Note $T_{\phi}$ is also $C_{\alpha, u, E_{\gamma}}$-symmetric. Using the similar arguments as we have done above, we obtain that $\gamma \beta_{\ell, j}=\alpha_{m} \overline{\alpha_{m+j}} \bar{\gamma} \eta_{\ell, j}$ and hence

$$
\beta_{\ell, j}=\bar{\gamma}^{2} \alpha_{m} \overline{\alpha_{m+j}} \eta_{\ell, j}
$$

for all $m \geq 0, \ell \geq 1$ and $j \geq 0$. Noting the above holds for any $\gamma \in \mathbb{T}$, we have $\beta_{\ell, j}=\eta_{\ell, j}=0$ for all $\ell \geq 1$ and $j \geq 0$. Hence we have

$$
\phi=\sum_{j=0}^{\infty}\left(a_{j} u^{j}+b_{j} \bar{u}^{j}\right)
$$

Also, using the equality

$$
\left\langle C_{\alpha, u, E} T_{\phi} C_{\alpha, u, E} u^{m}, u^{n}\right\rangle=\left\langle T_{\bar{\phi}} u^{m}, u^{n}\right\rangle,
$$

we have $\overline{\alpha_{m}} \alpha_{n} \overline{a_{n-m}}=\overline{b_{n-m}}$ for all $n>m \geq 0$, which implies

$$
b_{j}=\alpha_{m} \overline{\alpha_{m+j}} a_{j}, \quad j \geq 1, m \geq 0 .
$$

Since $\alpha \in \mathscr{M}$, we have $\left|b_{j}\right|=\left|a_{j}\right|$ for any $j \geq 1$ and then

$$
\frac{\alpha_{m+j}}{\alpha_{m}}=\frac{a_{j}}{b_{j}}
$$

for all $m \geq 0$ and $j \geq 1$ with $a_{j} \neq 0$. If $a_{j}=0$ for all $j \geq 1$, then clearly (c) holds. So, suppose $a_{j} \neq 0$ for some $j$. Put $N=\min \left\{j \geq 1: a_{j} \neq 0\right\}$ and $q=\frac{a_{N}}{b_{N}}$. Note $q \in \mathbb{T}$. By (2.3), we have $\frac{\alpha_{m+N}}{\alpha_{m}}=q$ for all $m \geq 0$, which yields

$$
\frac{\alpha_{m+N k}}{\alpha_{m}}=q^{k}, \quad m \geq 0, k \geq 1 .
$$

Now, in order to see that (c) holds, we consider the following two cases.

Case 1. Suppose $a_{N k} \neq 0$ for some $k \geq 1$. By (2.3) and (2.4), we have

$$
\frac{a_{N k}}{b_{N k}}=\frac{\alpha_{N k}}{\alpha_{0}}=q^{k}
$$

for all $k \geq 1$ with $a_{N k} \neq 0$. Hence $b_{N k}=\bar{q}^{k} a_{N k}$ for all $k \geq 1$. 
Case 2. Suppose $a_{N k+j} \neq 0$ for some $k \geq 1$ and $j$ with $1 \leq j<N$. By (2.3) and (2.4) again, we also see that

$$
\frac{a_{N k+j}}{b_{N k+j}}=\frac{\alpha_{N k+j+\ell}}{\alpha_{\ell}}=q^{k} \frac{\alpha_{j+\ell}}{\alpha_{\ell}}
$$

for $0 \leq \ell \leq N-j-1$ and

$$
\frac{a_{N k+j}}{b_{N k+j}}=\frac{\alpha_{N(k+1)+\ell-(N-j)}}{\alpha_{\ell}}=q^{k+1} \frac{\alpha_{\ell-(N-j)}}{\alpha_{\ell}}
$$

for $N-j \leq \ell<N$. It follows that

$$
\begin{aligned}
\frac{\alpha_{j}}{\alpha_{0}} & =\frac{\alpha_{j+1}}{\alpha_{1}}=\cdots=\frac{\alpha_{N-1}}{\alpha_{N-j-1}} \\
& =q \frac{\alpha_{0}}{\alpha_{N-j}}=q \frac{\alpha_{1}}{\alpha_{N-j+1}}=\cdots=q \frac{\alpha_{j-1}}{\alpha_{N-1}} .
\end{aligned}
$$

Multiplying all terms of the above together, we obtain

$$
\frac{\alpha_{j}}{\alpha_{0}}=q^{j / N}
$$

which gives

$$
\frac{a_{N k+j}}{b_{N k+j}}=q^{k} \frac{\alpha_{j}}{\alpha_{0}}=q^{(N k+j) / N} .
$$

Therefore, the above shows that $b_{N k+j}=\bar{q}^{(N k+j) / N} a_{N k+j}$ holds for any $a_{N k+j} \neq 0$, so does for any $a_{N k+j}$ with $k \geq 1$ and $1 \leq j<N$.

Noting $q \in \mathbb{T}$ and letting $\lambda=\bar{q}^{1 / N} \in \mathbb{T}$, we see from the two cases above, $b_{j}=\lambda^{j} a_{j}$ holds for all integers $j \geq 1$. Therefore we conclude that (c) holds with

$$
f=\frac{a_{0}+b_{0}}{2}+\sum_{j=1}^{\infty} a_{j} z^{j} \in H^{2} .
$$

The proof is complete.

Remark 2.2. We remark that it may not hold $\alpha_{j}=q^{j / N} \alpha_{0}$ for any $j$; see Example 2.6. We also note that the argument above proving $b_{j}=\lambda^{j} a_{j}$ is different from and much simpler than that in [2, Proposition 2.1] where canonical symmetric Toeplitz operators have been characterized.

Remark 2.3. Let $\phi \in L^{\infty}(\mathbb{T}), \lambda \in \mathbb{T}$ and $u$ be an inner function with $u(a)=0$ for some $a \in \mathbb{D}$. Choose an ONB $E=\left\{e_{a}, e_{1}, e_{2}, \ldots\right\}$ of

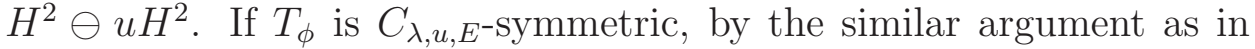
the proof of Theorem 1.1, we see that there are $f, g_{j} \in H^{2}(j \geq 1)$ such that

$$
\phi=f \circ u+\overline{C_{\lambda}(f) \circ u}+\sum_{j \geq 1}\left[\frac{e_{j}}{e_{a}} \cdot g_{j} \circ u+\overline{\frac{e_{j}}{e_{a}} \cdot C_{\lambda}\left(g_{j}\right) \circ u}\right] .
$$


Now we present examples asserting that $C_{\lambda, u, E^{-}}$symmetry of a Toeplitz operator generally depends on a choice of ONB $E$. In addition, the example also exhibits that a Toeplitz operator with the symbol $\phi$ appeared in the preceding remark may be complex symmetric.

Example 2.4. Given $a \in \mathbb{D} \backslash\{0\}$, we let $u=z \varphi_{a}$ and consider $E=\{1, e\}$ where $e=\gamma \frac{K_{a}-1}{\left\|K_{a}-1\right\|}$ and $\gamma \in \mathbb{T}$. Let $\lambda=\frac{a^{2}}{\bar{a}^{2}}$ and $\phi \in L^{\infty}(\mathbb{T})$. Using Theorem 1.1 and Remark 2.3, one can check that $T_{\phi}$ is $C_{\lambda, u, E}$-symmetric if and only if exactly one of the following statements holds:

(a) $\gamma \neq \pm 1$ and $\phi=f \circ u+\overline{C_{\lambda}(f) \circ u}$ for some $f \in H^{2}$.

(b) $\gamma= \pm 1$ and $\phi=f \circ u+\overline{C_{\lambda}(f) \circ u}+e(g \circ u)+\overline{e\left[C_{\lambda}(g) \circ u\right]}$ for some $f, g \in H^{2}$.

In the rest of this section, let's consider an inner function given by a single Möbius transform. To be more precise, given $a \in \mathbb{D}, \lambda \in \mathbb{T}$ and $\alpha \in \mathscr{M}$, let $e_{a}=\sqrt{1-|a|^{2}} K_{a}$. Consider $u=\varphi_{a}$ and $E_{\gamma}=\left\{\gamma e_{a}\right\}$ where $\gamma \in \mathbb{T}$. We put

$$
C_{\alpha, a}:=C_{\alpha, \varphi_{a}, E_{1}}, \quad C_{\lambda, a}:=C_{\lambda, \varphi_{a}, E_{1}}
$$

for notational simplicity. Since $C_{\alpha, \varphi_{a}, E_{\gamma}}=\gamma^{2} C_{\alpha, a}$, as an immediate consequence of Proposition 2.1 and Theorem 1.1, we have the following corollary which recovers known results in [2] and [13] in case $a=0$.

Corollary 2.5. Let $\phi \in L^{\infty}(\mathbb{T})$ and $a \in \mathbb{D}$. Then the following are equivalent:

(a) $T_{\phi}$ is $C_{\alpha, a}$-symmetric for some $\alpha \in \mathscr{M}$.

(b) $T_{\phi}$ is $C_{\lambda, a}$-symmetric for some $\lambda \in \mathbb{T}$.

(c) $T_{\phi \circ \varphi_{a}}$ is $C_{\lambda}$-symmetric for some $\lambda \in \mathbb{T}$.

(d) $\phi=f \circ \varphi_{a}+\overline{C_{\lambda}(f) \circ \varphi_{a}}$ for some $\lambda \in \mathbb{T}$ and $f \in H^{2}$.

(e) $\phi \circ \varphi_{a}(\lambda z)=\phi \circ \varphi_{a}(\bar{z})$ for all $z \in \mathbb{D}$ and some $\lambda \in \mathbb{T}$.

Given $a \in \mathbb{D}$ and $\lambda \in \mathbb{T}$, since

$$
U_{a} C_{\lambda} U_{a}\left(\varphi_{a}^{m} e_{a}\right)=\bar{\lambda}^{m} \varphi_{a}^{m} e_{a}=C_{\lambda, a}\left(\varphi_{a}^{m} e_{a}\right)
$$

for all integer $m \geq 0$, we have $C_{\lambda, a}=U_{a} C_{\lambda} U_{a}$ Using this fact, for $\phi \in L^{\infty}(\mathbb{T})$, we see that $T_{\phi}$ is $C_{\lambda, a}$-symmetric if and only if $U_{a} T_{\phi} U_{a}$ is

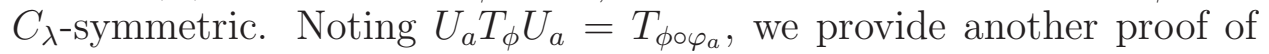
the equivalences of (b), (c), (d) and (e) of Corollary 2.5.

We give in passing an example of a Toeplitz operator which is complex symmetric with respect to $C_{\alpha, 0}$ and $C_{\lambda}$ for all $\lambda \in \mathbb{T}$ and some $\alpha \in \mathscr{M}$, although $C_{\alpha, 0} \neq \mu C_{\gamma, a}$ for any $a \in \mathbb{D} \backslash\{0\}$ and $\mu, \gamma \in \mathbb{T}$. 
Example 2.6. Let $\lambda \in \mathbb{T}$ and $\eta \in \mathbb{T} \backslash\{ \pm \bar{\lambda}\}$. Define a conjugation $C$ on $H^{2}$ by

$$
C z^{2 j}=\bar{\lambda}^{2 j} z^{2 j}, \quad C z^{2 j+1}=\eta \bar{\lambda}^{2 j} z^{2 j+1}
$$

for $j=0,1,2, \cdots$. So $C=C_{\alpha, 0}$ where

$$
\alpha=\left(1, \eta, \bar{\lambda}^{2}, \eta \bar{\lambda}^{2}, \bar{\lambda}^{4}, \eta \bar{\lambda}^{4}, \cdots\right) \in \mathscr{M} \text {. }
$$

Note $C \neq \mu C_{\gamma}$. We claim $C \neq \mu C_{\gamma, a}$ for any $a \in \mathbb{D} \backslash\{0\}$ and $\mu, \gamma \in$ $\mathbb{T}$. Suppose not. Then, $C=\mu C_{\gamma, a}$ for some $a, \mu, \gamma$. Then, a direct calculation shows

$$
C z^{n}=\mu \delta\left(\beta \varphi_{b}\right)^{n} e_{b}, \quad n=0,1,2, \cdots
$$

where $b=\varphi_{a}(\bar{a} \gamma), \delta=\frac{1-a^{2} \bar{\gamma}}{\left|1-a^{2} \bar{\gamma}\right|}$ and $\beta \in \mathbb{T}$ satisfying $\bar{b}=\beta b$. Comparing the above with the definition of $C$, we have $b=0$ and then $\gamma=a / \bar{a}$. Hence $C=\mu C_{-\bar{\beta}}$ and a contradiction follows. Then, for $\phi=z^{2}+z^{4}+$ $\lambda^{2} \bar{z}^{2}+\lambda^{4} \bar{z}^{4}$, it is clear that $T_{\phi}$ is complex symmetric with respect to both $C_{\lambda}$ and $C$.

We now provide another point of view for conjugations $C_{\lambda, a}$. Let $u: \mathbb{D} \rightarrow \mathbb{C}$ and $v: \mathbb{D} \rightarrow \mathbb{D}$ be two analytic functions. Define a conjugate-linear operator $\mathcal{A}_{u, v}$ on $H^{2}$ by

$$
\mathcal{A}_{u, v} f=u \overline{f(\bar{v})}
$$

for functions $f \in H^{2}$. Such conjugations are introduced in [15] where complex symmetric weighted composition operators have been studied. Clearly, if $u=\mu$ and $v=\bar{\lambda} z$ for some $\mu, \lambda \in \mathbb{T}$, then $\mathcal{A}_{u, v}=\mu C_{\lambda}$. So $\mathcal{A}_{u, v}$ is a generalization of the conjugations $\mu C_{\lambda}$.

We prove that an operator $\mathcal{A}_{u, v}$ being a conjugation is essentially the same as the conjugations $C_{\lambda, a}$ as shown in the following proposition, which is not only of own interest but also used in Proposition 4.4.

Proposition 2.7. Let $u: \mathbb{D} \rightarrow \mathbb{C}$ and $v: \mathbb{D} \rightarrow \mathbb{D}$ be two analytic functions. Then the following assertions are equivalent:

(a) $\mathcal{A}_{u, v}$ is a conjugation on $H^{2}$.

(b) $u=\alpha e_{b}$ and $v=\beta \varphi_{b}$ for some $\alpha, \beta \in \mathbb{T}$ and $b \in \mathbb{D}$ with $\bar{b}=\beta b$.

(c) $\mathcal{A}_{u, v}=\mu C_{\lambda, a}$ for some $\mu, \lambda \in \mathbb{T}$ and $a \in \mathbb{D}$.

Proof. The equivalence of (a) and (b) has been proved in 15. Now assume (c) and show (b). If $a=0$, then (b) holds with $u=\mu$ and $v=\bar{\lambda} z$. So, suppose $a \neq 0$. If $\lambda \neq a / \bar{a}$, then a straightforward calculation yields $\overline{e_{a}\left(\lambda \overline{\varphi_{a}}\right)} e_{a}=\bar{\gamma} e_{\varphi_{a}(\bar{a} \lambda)}$ and

$$
\overline{\varphi_{a}\left(\lambda \overline{\varphi_{a}}\right)}=\varphi_{\overline{a^{2}}}(\bar{\lambda}) \varphi_{\varphi_{a}(\bar{a} \lambda)}=(\bar{b} / b) \varphi_{b}
$$


where

$$
b=\varphi_{a}(\bar{a} \lambda), \quad \gamma=\frac{1-a^{2} \bar{\lambda}}{\left|1-a^{2} \bar{\lambda}\right|}
$$

for simplicity. It follows that

$$
\begin{aligned}
C_{\lambda, a} z^{n} & =U_{a} C_{\lambda} U_{a} z^{n} \\
& =\overline{\left[\varphi_{a}\left(\lambda \overline{\varphi_{a}}\right)\right]^{n} e_{a}\left(\lambda \overline{\varphi_{a}}\right)} e_{a} \\
& =\bar{\gamma}\left[(\bar{b} / b) \varphi_{b}\right]^{n} e_{b}
\end{aligned}
$$

for all integer $n \geq 0$. Therefore, we have

$$
C_{\lambda, a} f=\bar{\gamma} e_{b} \overline{f\left(\overline{\left.(\bar{b} / b) \varphi_{b}\right)}\right.}
$$

for all $f \in H^{2}$. So (b) holds with $u=\mu \bar{\gamma} e_{b}$ and $v=(\bar{b} / b) \varphi_{b}$. If $\lambda=a / \bar{a}$, it follows from (2.6) ) that $C_{\lambda, a} z^{n}=(\bar{\lambda} z)^{n}$ for all integer $n \geq 0$. Thus $C_{\lambda, a} f=\overline{f(\overline{\bar{\lambda} z})}$ for all $f \in H^{2}$ and (b) follows with $u=\mu$ and $v=\bar{\lambda} z$.

Finally assume (b) and show (c). If $b=0$, then it is obvious that $\mathcal{A}_{u, v}=\alpha C_{-\bar{\beta}}$. Now suppose $b \neq 0$. It is routine to see that there exist $\lambda \in \mathbb{T}$ and $a \in \mathbb{D}$ such that $b=\varphi_{a}(\bar{a} \lambda)$. Put $\gamma:=\frac{1-a^{2} \bar{\lambda}}{\left|1-a^{2} \bar{\lambda}\right|}$. Then, (2.6) shows that

$$
C_{\lambda, a} z^{n}=\bar{\gamma}\left(\beta \varphi_{b}\right)^{n} e_{b}, \quad n=0,1,2, \cdots
$$

because $\beta=\bar{b} / b=\varphi \overline{a^{2}}(\bar{\lambda})$. Thus $\mathcal{A}_{u, v}=\alpha \gamma C_{\lambda, a}$ and (c) holds. The proof is complete.

\section{A Class of uet Toeplitz operators}

In this section, we first show that Question 1.2 is true for certain Toeplitz operators induced by a Möbius transform. We also prove Theorem 1.3 .

A conjugate-linear map $D$ on a Hilbert space $\mathcal{H}$ is called anti-unitary if $D$ is invertible and isometric. Clearly, a conjugation is anti-unitary. Recall that an operator $T$ on $\mathcal{H}$ is called UET if $T \cong C T^{*} C$ for some conjugation $C$ on $\mathcal{H}$. The following lemma shows that UET operators can be characterized by anti-unitary operators; see [8] for details.

Lemma 3.1. For a bounded linear operator $T$ on $\mathcal{H}, T$ is UET if and only if $D T D^{-1}=T^{*}$ for some anti-unitary operator $D$ on $\mathcal{H}$.

We introduce nowhere winding curves which are closely related to complex symmetric/UET Toeplitz operators. For a closed curve $\gamma$ in $\mathbb{C}$, the winding number of $\gamma$ with respect to $z \in \mathbb{C} \backslash \gamma$, also called the index of $\gamma$, is defined by

$$
\operatorname{Ind}_{\gamma}(z)=\frac{1}{2 \pi i} \int_{\gamma} \frac{1}{\zeta-z} d \zeta, \quad z \in \mathbb{C} \backslash \gamma .
$$


Recall that the curve $\gamma$ is called nowhere winding if $\operatorname{Ind}_{\gamma}(z)=0$ for all $z \in \mathbb{C} \backslash \gamma$. Given $\phi \in L^{\infty}(\mathbb{T})$, a well known Coburn theorem says that at least one of $T_{\phi}$ and $T_{\phi}^{*}$ is injective; see [3] or [5] for details. Using this fact together with the Fredholm index theorem([5]), Noor [17] recently proved that the curve $\phi(\mathbb{T})$ of the continuous symbol $\phi$ of a complex symmetric Toeplitz operator $T_{\phi}$ is nowhere winding. Note each complex symmetric operator is an UET operator. By using a similar argument as in [17], we can see that the same is true for UET operators as shown in the following.

Proposition 3.2. Let $\phi$ be a continuous function on $\mathbb{T}$. If $T_{\phi}$ is UET, then $\phi(\mathbb{T})$ is a nowhere winding curve.

In [17, Noor posed a question: Does every nowhere winding curve induce a complex symmetric operator? In view of Proposition [3.2, it is natural to ask the same question for UET operators. The following provides a positive answer for certain Toeplitz operators.

Proposition 3.3. For $q \in \mathbb{T}$ and $b \in \mathbb{D}$, let $\phi=z+\overline{q \varphi_{b}}$. Then the following statements are equivalent:

(a) $T_{\phi}$ is complex symmetric.

(b) $T_{\phi}$ is UET.

(c) $T_{\phi}$ is either normal or $C_{\lambda, a}$-symmetric for some $\lambda \in \mathbb{T}$ and $a \in \mathbb{D}$.

(d) The curve $\phi(\mathbb{T})$ is nowhere winding.

(e) $q b=\bar{b}$.

Proof. Since implications $(\mathrm{c}) \Longrightarrow(\mathrm{a}) \Longrightarrow(\mathrm{b})$ is obvious and $(\mathrm{b}) \Longrightarrow$ (d) follows from Proposition 3.2, we only need to prove implications (d) $\Longrightarrow(\mathrm{e})$ and $(\mathrm{e}) \Longrightarrow(\mathrm{c})$. First assume (d) and show (e). Put $s:=\bar{q} \bar{b}-b$. By a direct computation, we see

$$
\phi(z)-s=\frac{z^{2}-\left[\bar{q}\left(1-|b|^{2}\right)+b^{2}\right]}{z-b}, \quad z \in \mathbb{T} .
$$

If $q b \neq \bar{b}$, a routine calculation shows $\left|\bar{q}\left(1-|b|^{2}\right)+b^{2}\right|<1$, which implies that $s \notin \phi(\mathbb{T})$ and two zeros of $z^{2}-\left[\bar{q}\left(1-|b|^{2}\right)+b^{2}\right]$ are all in $\mathbb{D}$. Hence, by the argument principle, we see that

$$
\operatorname{Ind}_{\phi(\mathbb{T})}(s)=\frac{1}{2 \pi i} \int_{\phi(\mathbb{T})} \frac{1}{\zeta-s} d \zeta=\frac{1}{2 \pi i} \int_{\mathbb{T}} \frac{\phi^{\prime}(\zeta)}{\phi(\zeta)-s} d \zeta=1,
$$

which is a contradiction and $(\mathrm{d}) \Longrightarrow(\mathrm{e})$ holds.

Finally, assume (e) and show (c). If $b=0$, then $\phi=z-\overline{q z}$ and $T_{\phi}$ is a normal operator. Now suppose $b \neq 0$. It is routine to see that there 
exist $a \in \mathbb{D}$ and $\lambda \in \mathbb{T}$ such that $b=\varphi_{a}(\bar{a} \lambda)$. Then, using applications of (2.1) and (2.5), we can check that

$$
C_{\lambda, a} T_{z+\overline{q \varphi_{b}}} C_{\lambda, a}=T_{C_{\lambda}\left(\varphi_{a}+\overline{q \varphi_{b} \circ \varphi_{a}}\right) \circ \varphi_{a}}=T_{z+\overline{q \varphi_{b}}}^{*},
$$

thus $T_{z+\overline{q \varphi_{b}}}$ is $C_{\lambda, a^{-}}$-symmetric and (c) follows, as desired.

Given an operator $T$, we let $\operatorname{ran} T$, $\operatorname{ker} T$ and $\operatorname{tr} T$ denote the range, kernel and trace of $T$ respectively. Also, let $[A, B]=A B-B A$ denote the commutator of two operators $A$ and $B$.

The following lemma will be useful in the proof of Theorem 1.3. Given an inner function $u$, we denote ord $u=\operatorname{dim} H^{2} \ominus u H^{2}$.

Lemma 3.4. Let $f, g$ be finite Blaschke products and $\mu \in \mathbb{C}$. Put $\phi=f+\mu \bar{g}$. If $D T_{\phi}=T_{\phi}^{*} D$ for some anti-unitary operator $D$ on $H^{2}$, then the following statements hold:

(a) $\left[T_{\phi}^{*}, T_{\phi}\right]$ has finite rank and $\operatorname{ran}\left[T_{\phi}^{*}, T_{\phi}\right]$ reduces $D$.

(b) ord $f=|\mu|^{2}$ ord $g$.

Proof. Set $T=\left[T_{\phi}^{*}, T_{\phi}\right]$. It is easy to check

$$
T=\left[T_{f}^{*}, T_{f}\right]-|\mu|^{2}\left[T_{g}^{*}, T_{g}\right] .
$$

Since $T_{f}$ is an isometry, $\left[T_{f}^{*}, T_{f}\right]=I-T_{f} T_{f}^{*}$ is a projection onto $H^{2} \ominus$ $f H^{2}$ and hence

$$
\operatorname{tr}\left[T_{f}^{*}, T_{f}\right]=\operatorname{dim}\left(H^{2} \ominus f H^{2}\right)=\operatorname{ord} f .
$$

Similarly, we have $\operatorname{tr}\left[T_{g}^{*}, T_{g}\right]=$ ord $g$. Thus $T$ is a finite-rank operator and $\operatorname{tr} T=$ ord $f-|\mu|^{2}$ ord $g$. Also, since $D T_{\phi}=T_{\phi}^{*} D$, it follows that $D T=-T D$. Thus $D(\operatorname{ker} T) \subset \operatorname{ker} T$ and $D(\operatorname{ran} T) \subset \operatorname{ran} T$. Noting that $T$ is self-adjoint, we see that $\operatorname{ker} T \oplus \operatorname{ran} T=H^{2}$ and hence $\operatorname{ran} T$ reduces $D$. This gives (a).

The condition $D T_{\phi}=T_{\phi}^{*} D$ also yields $D T D^{-1}=-T$. It follows that $\operatorname{tr} T=0$, which implies (b). The proof is complete.

Now we are ready to prove Theorem 1.3 .

Proof of Theorem 1.3. Set $\psi:=z+\overline{\mu B_{2}}$ and then $\phi=\psi \circ B_{1}$. Let ord $B_{1}=m$.

First assume (b) and show (c). Since $T_{\phi} \cong T_{\psi}^{(m)}$ (see [4]) and $T_{\psi}$ is essentially normal, by the similar argument as in the proof of Theorem 1.4 of [2], we see $T_{\psi}$ is also UET. By Proposition [3.3, it suffices to prove that $\mu \in \mathbb{T}$ and ord $B_{2}=1$. Suppose $D^{-1} T_{\psi} D=T_{\psi}^{*}$ for some anti-unitary $D$ on $H^{2}$. By (3.1) and Lemma 3.4(b), we have

$$
T:=\left[T_{\psi}^{*}, T_{\psi}\right]=\left(I-T_{z} T_{\bar{z}}\right)-|\mu|^{2}\left(I-T_{B_{2}} T_{\bar{B}_{2}}\right)
$$


and $1=|\mu|^{2}$ ord $B_{2}$, which means that $0<|\mu| \leq 1$. If $|\mu|=1$, then ord $B_{2}=1$, as desired. So, suppose $|\mu|<1$ and put $\mathscr{R}=\operatorname{ran} T$. Noting that $I-T_{z} T_{\bar{z}}$ is a projection onto $\mathbb{C}$ and $I-T_{B_{2}} T_{\bar{B}_{2}}$ is a projection onto $H^{2} \ominus B_{2} H^{2}$, we have

$$
\mathscr{R} \subset \vee\left\{1, H^{2} \ominus B_{2} H^{2}\right\} .
$$

We claim $1 \in \mathscr{R}$. In fact, if $B_{2}(0)=0$, then $T 1=1-|\mu|^{2}$; if $B_{2}(0) \neq 0$, then $T B_{2}=B_{2}(0)$, which proves the claim. Now, by Lemma 3.4(a), we know that $D(\mathscr{R}) \subset \mathscr{R}$. Since $1 \in \mathscr{R}$, in view of (3.2), we may assume $D 1=\alpha+h$ for some $\alpha \in \mathbb{C}$ and $h \in H^{2} \ominus B_{2} H^{2}$. Recalling $T_{\psi}^{*} 1=D^{-1} T_{\psi} D 1$ and noting $H^{2} \ominus B_{2} H^{2}=\operatorname{ker} T_{\bar{B}_{2}}$, we obtain

$$
\mu B_{2}=T_{\psi}^{*} 1=D^{-1}\left[z D 1+\bar{\mu} \alpha \overline{B_{2}(0)}\right]
$$

which implies

$$
|\mu|^{2}=\left\|\mu B_{2}\right\|^{2}=\| z D 1+\left.\bar{\mu} \alpha \overline{B_{2}(0)}\right|^{2}=1+\left|\mu \alpha B_{2}(0)\right|^{2} .
$$

So we have

$$
1=|\mu|^{2}\left(1-|\alpha|^{2}\left|B_{2}(0)\right|^{2}\right)
$$

which contradicts the hypothesis $|\mu|<1$. Then we conclude that $|\mu|=1$ and ord $B_{2}=1$ as desired.

Now assume (c). By Proposition 3.3. $T_{\psi}$ is complex symmetric, then so is $T_{\phi}$ because $T_{\phi} \cong T_{\psi}^{(m)}$. Hence (a) holds. Since implication $(\mathrm{a}) \Longrightarrow(\mathrm{b})$ is obvious, we complete the proof.

\section{Analytic symmetry of Toeplitz operators}

In this section, we first show that an analytic symmetric Toeplitz operator with trigonometric symbol is $C_{\lambda}$-symmetric for some $\lambda \in \mathbb{T}$. Also, we characterize certain Toeplitz operators to be analytically symmetric.

Given $\phi \in L^{\infty}(\mathbb{T})$, we recall that $T_{\phi}$ is analytically symmetric if there exist $f, g \in H^{\infty}$ and a conjugation $C$ on $H^{2}$ such that $C T_{f} C=T_{g}$ and $\phi=f+\bar{g}$. Also, analytic symmetric Toeplitz operators are complex symmetric.

To begin with, for $f \in H^{\infty}$, inner $u$ and $\lambda \in \mathbb{T}$, we first recall $C_{\lambda} f(z)=\overline{f(\lambda \bar{z})}$ and note from (2.1) that the Toeplitz operator of form

$$
T_{f \circ u+\overline{C_{\lambda}(f) \circ u}}=T_{f \circ u+\overline{C_{1}(f) \circ(\bar{\lambda} u)}}
$$

is analytically symmetric. The following lemma shows that any analytic symmetric Toeplitz operator has the similar form above under certain condition. 
Lemma 4.1. Let $f, g \in H^{\infty}$ and $\alpha \in \mathbb{C}$. Suppose the inner factor of $f-\alpha$ is a nonconstant finite Blaschke product. If $C T_{f} C=T_{g}$ for some conjugation $C$, then there exist $h \in H^{\infty}$ and finite Blaschke products $u, u_{1}$ with ord $u=\operatorname{ord} u_{1}$ such that $f=h \circ u$ and $g=C_{1} h \circ u_{1}$.

Proof. Put $U=C C_{1}$. Then, it is easy to see that $U$ is a unitary operator and

$$
U^{*} T_{f} U=C_{1} C T_{f} C C_{1}=C_{1} T_{g} C_{1}=T_{C_{1} g}
$$

on $H^{2}$. It follows from [4, Corollary 1] that there exist $h \in H^{\infty}$ and two finite Blaschke products $u, u_{2}$ with ord $u=\operatorname{ord} u_{2}$ such that $f=h \circ u$ and $C_{1} g=h \circ u_{2}$, which immediately implies the desired result by taking $u_{1}=C_{1} u_{2}$. We complete the proof.

For trigonometric symbols $\phi$, we show below that Toeplitz operator $T_{\phi}$ is analytically symmetric only when $\phi=f+\overline{C_{\lambda} f}$ where $f$ is an analytic polynomial.

Theorem 4.2. Let $\phi$ be a trigonometric polynomial. Then $T_{\phi}$ is analytically symmetric if and only if $T_{\phi}$ is $C_{\lambda}$-symmetric for some $\lambda \in \mathbb{T}$.

Proof. By Proposition 2.1, the sufficiency is clear because $\phi=f+\overline{C_{\lambda} f}$ and $C_{\lambda} T_{f} C_{\lambda}=T_{C_{\lambda} f}$ for all $\lambda \in \mathbb{T}$ and $f \in H^{\infty}$.

Now we prove the necessity. Suppose $\phi=f+\bar{g}$ and $C T_{f} C=T_{g}$ for some conjugation $C$ and analytic polynomials $f, g$. Since $f-\alpha$ is still an analytic polynomial for any $\alpha \in \mathbb{C}$, its inner factor is a finite Blaschke product. Thus by Lemma 4.1, there exist $h \in H^{\infty}$ and two finite Blaschke products $u$ and $u_{1}$ with ord $u=$ ord $u_{1}$ such that

$$
f=h \circ u, \quad g=\left(C_{1} h\right) \circ u_{1} .
$$

Since $f$ and $g$ are both analytic polynomials, we may assume that $h$ is an analytic polynomial and $u=\beta z^{N}, u_{1}=\beta_{1} z^{N}$ where $\beta, \beta_{1} \in \mathbb{T}$ and $N$ is a positive integer. Hence

$$
f+\bar{g}=h \circ\left(\beta z^{N}\right)+\overline{C_{1}(h) \circ\left(\beta_{1} z^{N}\right)}=h \circ u+\overline{C_{\lambda}(h) \circ u},
$$

where $\lambda=\beta \overline{\beta_{1}}$. Thus by Proposition 2.1. $T_{f+\bar{g}}$ is $C_{\lambda, u, E^{-} \text {-symmetric }}$ where $E=\left\{1, z, z^{2}, \cdots, z^{N-1}\right\}$. It follows from the definition of $C_{\lambda, u, E}$ that

$$
C_{\lambda, u, E}\left(u^{m} z^{j}\right)=\bar{\lambda}^{m} u^{m} z^{j}, \quad m \geq 0, j=0,1, \cdots, N-1 .
$$

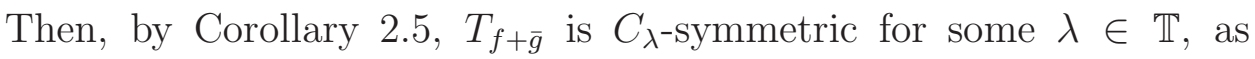
desired. The proof is complete.

We provide an example of a complex symmetric Toeplitz operator which is not analytically symmetry. 
Example 4.3. Let $\phi=z+z^{2}+i\left(\overline{z+z^{2}}\right)$. Then $T_{\phi}$ is obviously normal and hence complex symmetric. However, by Proposition 2.1(f), we

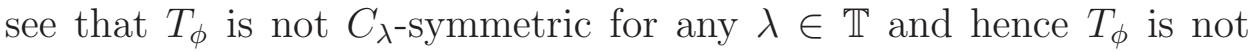
analytically symmetric by Theorem 4.2 .

In the following theorem, we characterize an analytic symmetric Toeplitz operator when the analytic part of its symbol is a polynomial of degree 2 . We were not able to characterize general symbols.

Theorem 4.4. Let $\ell, c, d \in \mathbb{C}$ and $g \in H^{\infty}$ with $g(0)=0$. Put $p=$ $d z+c z^{2}$ and $\phi=\ell+p+\bar{g}$. Then the following statement are equivalent:

(a) $T_{\phi}$ is analytically symmetric on $H^{2}$.

(b) There exist $q \in \mathbb{T}$ and $b \in \mathbb{D}$ with $\bar{b}=q b$ such that one of the following statements holds:

(b1) $d \neq 0$ and $g=\bar{d} q \varphi_{b}+\bar{c}\left(q \varphi_{b}\right)^{2}$.

(b2) $d=0$ and $g=\bar{c}\left(q \varphi_{b}\right)^{2}$.

(b3) $d=0$ and $g=\bar{c} q \varphi_{b}\left(z^{2}\right)$.

Proof. We first assume (b) and prove (a). By (2.5), we note $C_{\lambda}\left(\varphi_{a}\right) \circ$ $\varphi_{a}=q \varphi_{b}$ where $\bar{b}=q b$ with $b=\varphi_{a}(\bar{a} \lambda)$. It follows from (2.1) that

$$
C_{\lambda, a} T_{p} C_{\lambda, a}=T_{C_{\lambda}\left(p \circ \varphi_{a}\right) \circ \varphi_{a}}=T \overline{p\left(\bar{q} \overline{\varphi_{b}}\right)}
$$

and

$$
C_{\lambda, \varphi_{a} \circ z^{2}, E} T_{z^{2}} C_{\lambda, \varphi_{a} \circ z^{2}, E}=T_{C_{\lambda}\left(\varphi_{a}\right) \circ \varphi_{a} \circ z^{2}}=T_{q \varphi_{b} \circ z^{2}}
$$

for an ONB $E$ of the model space $H^{2} \ominus\left(\varphi_{a} \circ z^{2}\right) H^{2}$. Hence Toeplitz operators $T_{p+p\left(\bar{q} \overline{\varphi_{b}}\right)}$ and $T_{z^{2}+\overline{q \varphi_{b} \circ z^{2}}}$ with $\bar{b}=q b$ both are analytically symmetric and (a) holds.

Now we assume (a) and prove (b). We suppose that

$$
C T_{p+\ell_{1}} C=T_{g+\bar{\ell}_{2}}
$$

for some conjugation $C$, here $\ell_{1}+\ell_{2}=\ell$. Since $p+\ell_{1}$ is an analytic polynomial, by Lemma 4.1, one may assume

$$
g+\bar{\ell}_{2}=C_{1}\left(p+\ell_{1}\right) \circ B=\bar{\ell}_{1}+C_{1}(p) \circ B
$$

for some finite Blaschke product $B$ with $\operatorname{ord} B=1$ or 2 according to $\ell_{1}, d$ and $c$. Thus (4.1) becomes $C T_{p} C=T_{g}$ and $g=C_{1}(p) \circ B$. Hence we easily see

$$
C\left(\operatorname{ker} T_{p-\beta}^{*}\right)=\operatorname{ker} T_{g-\bar{\beta}}^{*}
$$

for any $\beta \in \mathbb{C}$.

Now we consider several cases. First suppose $d \neq 0$ and $c \neq 0$. In this case, we may take $B=q \varphi_{b}$ for some $q \in \mathbb{T}$ and $b \in \mathbb{D}$ and hence $g=\bar{d} q \varphi_{b}+\bar{c}\left(q \varphi_{b}\right)^{2}$. Put

$$
E_{d}=\{z \in \mathbb{D}:|(d / c)+z|>1\} .
$$


Then $E_{d}$ is a nonempty open set in $\mathbb{D}$ because $d / c \neq 0$. Let $z \in E_{d}$ be any point. Then we note

$$
\operatorname{ker} T_{p-\beta}^{*}=\mathbb{C} \cdot K_{z}, \quad \operatorname{ker} T_{g-\bar{\beta}}^{*}=\mathbb{C} \cdot K_{\varphi_{b}(\bar{q} \bar{z})}
$$

where $\beta:=d z+c z^{2}$. Hence, by (4.2), one can see that there is $\eta(z) \in \mathbb{C}$ such that $C K_{z}=\eta(z) K_{\varphi_{b}(\bar{q} \bar{z})}$. It follows that

$$
C h(z)=\left\langle C h, K_{z}\right\rangle=\left\langle C K_{z}, h\right\rangle=\eta(z) \overline{h\left(\overline{q \varphi_{\bar{q} \bar{b}}(z)}\right)}
$$

for every $h \in H^{2}$. By considering $h=1$, we have $\eta=C 1$ and hence

$$
C h(z)=C 1(z) \overline{h\left(\overline{q \varphi_{\bar{q} \bar{b}}(z)}\right)}, \quad h \in H^{2}
$$

for each $z \in E_{d}$. Because $E_{d}$ is open in $\mathbb{D}$, the above holds for any $z \in \mathbb{D}$. Therefore, an application of Proposition 2.7 shows $\bar{b}=q b$ and then (a) holds.

If $d \neq 0$ and $c=0$, one can see that (a) holds by the similar proof.

Now, suppose $d=0$ and assume $p=z^{2}$ without loss of generality. As before we may further assume $g=\left(q \varphi_{b}\right)^{2}$ or $g=q \varphi_{a} \varphi_{b}$, where $q \in \mathbb{T}$ and $a, b \in \mathbb{D}$ with $a \neq b$. We divide the proof into two cases below.

Case 1. Consider $g=\left(q \varphi_{b}\right)^{2}$. Since the case $b=0$ is trivial, we assume $b \neq 0$. Taking $\beta=0$ in (4.2) we have

$$
C 1=u_{1} K_{b}+v_{1} \partial_{\bar{b}} K_{b}
$$

for some $u_{1}, v_{1} \in \mathbb{C}$ where $\partial_{\bar{b}} K_{b}(z)=z(1-\bar{b} z)^{-2}$. Again, taking $\bar{\beta}=$ $q^{2} b^{2}$ in $(4.2)$ we see $1 \in \operatorname{ker} T_{g-\bar{\beta}}^{*}$ and $K_{\bar{q} \bar{b}}, K_{-\bar{q} \bar{b}} \in \operatorname{ker} T_{p-\beta}^{*}$, so there are $u_{2}, v_{2} \in \mathbb{C}$ such that

$$
C 1=u_{2} K_{\bar{q} \bar{b}}+v_{2} K_{-\bar{q} \bar{b}} .
$$

Comparing the above with (4.3), we obtain $b=\bar{q} \bar{b}$ or $b=-\bar{q} \bar{b}$. Hence (b) follows, as desired.

Case 2. Consider $g=q \varphi_{a} \varphi_{b}$ with $a \neq b$. We first assume $a b \neq 0$. Taking $\beta=0$ in the equality (4.2) we have

$$
C 1=u_{1} K_{a}+v_{1} K_{b}, \quad C z=u_{2} K_{a}+v_{2} K_{b}
$$

for some $u_{j}, v_{j} \in \mathbb{C}, j=1,2$. Taking $\bar{\beta}=q a b=$ : $\bar{\alpha}^{2}$, then $1 \in \operatorname{ker} T_{g-\bar{\beta}}^{*}$ and $K_{\alpha}, K_{-\alpha} \in \operatorname{ker} T_{p-\beta}^{*}$. So there are $u_{3}, v_{3} \in \mathbb{C}$ such that

$$
C 1=u_{3} K_{\alpha}+v_{3} K_{-\alpha} .
$$

Comparing the above with $C 1$ in (4.4), we may assume $\alpha=a$ without loss of generality, and hence $\bar{a}^{2}=q a b$ and $u_{1}=u_{3}$. Now we will show $b=-a$. Then we have $g=q \varphi_{a} \varphi_{-a}=-q \varphi_{a^{2}} \circ z^{2}$ and (b) holds. If 
$v_{1} \neq 0$ or $v_{3} \neq 0$, then we get $b=-\alpha=-a$, as desired. Now we suppose $v_{1}=v_{3}=0$. Hence (4.4) becomes

$$
C 1=u_{1} K_{a}, \quad C z=u_{2} K_{a}+v_{2} K_{b} .
$$

Since $C T_{z^{2}}\left(z^{n}\right)=T_{g} C\left(z^{n}\right)$ for all integer $n \geq 0$, it follows that

$$
C z^{2 n}=g^{n} u_{1} K_{a}, \quad C z^{2 n+1}=g^{n}\left(u_{2} K_{a}+v_{2} K_{b}\right)
$$

for all integer $n \geq 0$. Put $W=C C_{1}$. Then $W$ is unitary and $C_{1} W C_{1}=$ $W^{*}$. Also,

$$
W z^{2 n}=g^{n} u_{1} K_{a}, \quad W z^{2 n+1}=g^{n}\left(u_{2} K_{a}+v_{2} K_{b}\right)
$$

for all integer $n \geq 0$. So, for each $h \in H^{2}$ with $h(z)=\sum_{n=0}^{\infty} a_{n} z^{n}$, we have

$$
\begin{aligned}
W h(z) & =\sum_{n=0}^{\infty} a_{2 n} W\left(z^{2 n}\right)+\sum_{n=0}^{\infty} a_{2 n+1} W\left(z^{2 n+1}\right) \\
& =u_{1} K_{a} \sum_{n=0}^{\infty} a_{2 n} g^{n}+\left(u_{2} K_{a}+v_{2} K_{b}\right) \sum_{n=0}^{\infty} a_{2 n+1} g^{n} \\
& =U_{1}(z) h\left(V_{1}(z)\right)+U_{2}(z) h\left(V_{2}(z)\right)
\end{aligned}
$$

for $z$ in a connected open set $E \subset \mathbb{D}-\{a, b\}$ where

$$
\begin{array}{cc}
U_{1}=\frac{1}{2}\left(u_{1} K_{a}+\frac{u_{2} K_{a}+v_{2} K_{b}}{\sqrt{g}}\right), & V_{1}=\sqrt{g}, \\
U_{2}=\frac{1}{2}\left(u_{1} K_{a}-\frac{u_{2} K_{a}+v_{2} K_{b}}{\sqrt{g}}\right), & V_{2}=-\sqrt{g} .
\end{array}
$$

Note

$$
\left\langle W^{*} K_{z}, h\right\rangle=\left\langle K_{z}, W h\right\rangle=\left\langle\overline{U_{1}(z)} K_{V_{1}(z)}+\overline{U_{2}(z)} K_{V_{2}(z)}, h\right\rangle
$$

for each $z \in E$ and $h \in H^{2}$. Thus

$$
W^{*} K_{z}=\overline{U_{1}(z)} K_{V_{1}(z)}+\overline{U_{2}(z)} K_{V_{2}(z)}, \quad z \in E .
$$

Since $W C_{1} K_{z}=C_{1} W^{*} K_{z}$ for $z \in E$, we get

$$
U_{1} \cdot K_{\bar{z}} \circ V_{1}+U_{2} \cdot K_{\bar{z}} \circ V_{2}=U_{1}(z) K_{\overline{V_{1}(z)}}+U_{2}(z) K_{\overline{V_{2}(z)}}
$$

and hence

$$
\frac{U_{1}(w)}{1-z V_{1}(w)}+\frac{U_{2}(w)}{1-z V_{2}(w)}=\frac{U_{1}(z)}{1-V_{1}(z) w}+\frac{U_{2}(z)}{1-V_{2}(z) w}
$$

for all $z \in E$ and $w \in \mathbb{D}$. By direct computations, we see that (4.6) yields the following two equalities:

$$
\begin{aligned}
u_{1} \bar{b} q(a+b) & =\left(u_{2} \bar{b}+v_{2} \bar{a}\right)(\bar{a} \bar{b}+q a b), \\
u_{1} q(a+b)+u_{1} \bar{a} \bar{b}^{2} & =\left(u_{2}+v_{2}\right) q a b+\left(u_{2} \bar{b}+v_{2} \bar{a}\right)(\bar{a}+\bar{b}) .
\end{aligned}
$$


On the other hand, since $\langle C 1, C z\rangle=0$ and $\langle C z, 1\rangle=\langle C 1, z\rangle$, we have

$$
u_{2}+v_{2}=a\left(u_{2} \bar{b}+v_{2} \bar{a}\right)=u_{1} \bar{a} .
$$

Recall $q a b=\bar{a}^{2}$. It follows from (4.7) and (4.8) that

$$
\begin{aligned}
\bar{b} q(a+b) & =a^{-1} \bar{a}^{2}(\bar{a}+\bar{b}), \\
q(a+b) & =\bar{a}\left(\bar{a}^{2}-\bar{b}^{2}\right)+a^{-1} \bar{a}(\bar{a}+\bar{b}) .
\end{aligned}
$$

Therefore, we have $q(a+b)=\bar{a}^{2}(\bar{a}+\bar{b})$ and then $a+b=0$ as desired. For the case $a=0($ resp. $b=0)$, we can also see $b=0$ (resp. $a=0)$ by the similar argument. We omit the detail and finish the proof.

We close the paper with a characterization of conjugations $C$ on $H^{2}$ for which

$$
f \in H^{\infty} \Longrightarrow C T_{f} C \in\left\{T_{g}: g \in H^{\infty}\right\} .
$$

The notation $\mathcal{B}$ denotes the algebra of all bounded linear operators on $H^{2}$. Also, a Toeplitz operator is called an analytic Toeplitz operator if its symbol is analytic.

Theorem 4.5. Let $C$ be a conjugation on $H^{2}$ and $\Phi$ be a conjugatelinear map on $\mathcal{B}$ defined by $\Phi(L)=C L C$ for $L \in \mathcal{B}$. Then the following statements are equivalent:

(a) $\Phi$ leaves the collection of analytic Toeplitz operators invariant.

(b) $C=\gamma C_{\lambda, a}$ for some $\gamma, \lambda \in \mathbb{T}$ and $a \in \mathbb{D}$.

Proof. First assume (b). By (2.1), we have $C T_{f} C=T_{C_{\lambda}\left(f \circ \varphi_{a}\right) \circ \varphi_{a}}$ for all $f \in H^{\infty}$ and hence (a) holds.

Now assume (a). Then, for each $f \in H^{\infty}$, there exists $A f \in H^{\infty}$ such that

$$
C T_{f} C=T_{A f} \quad \text { or } \quad T_{f}=C T_{A f} C .
$$

Clearly, such $A f$ is unique. This induces a conjugate-linear map $A$ : $H^{\infty} \rightarrow H^{\infty}$. For each $f \in H^{\infty}$, note that

$$
T_{f}=C T_{A f} C=C\left(C T_{A A f} C\right) C=T_{A A f} .
$$

Hence $A^{2} f=f$ for all $f \in H^{2}$ and then $A^{2}=I$. Also, for $f, g \in H^{\infty}$, we note

$$
\begin{aligned}
T_{A(f g)} & =C T_{f g} C=C T_{f} T_{g} C \\
& =\left(C T_{f} C\right)\left(C T_{g} C\right)=T_{A f} T_{A g}=T_{A f \cdot A g},
\end{aligned}
$$

which deduce that

$$
A(f g)=A f \cdot A g .
$$

On the other hand, since $T_{f} C=C T_{A f}$, we have

$$
f \cdot C 1=T_{f} C 1=C T_{A f} 1=C(A f \cdot 1)=C A f
$$


and hence $A f=C(f \cdot C 1)$ for all $f \in H^{\infty}$. Hence we get

$$
C T_{f} C=T_{C(f \cdot C 1)}
$$

for all $f \in H^{\infty}$. Taking $f=z^{n}$ and using (4.9), we get

$$
T_{[C(z \cdot C 1)]^{n}}=T_{(A z)^{n}}=T_{A z^{n}}=C T_{z^{n}} C=T_{C\left(z^{n} \cdot C 1\right)}
$$

for all $n=0,1,2, \cdots$. Also, taking $n=1$ above and using Lemma 4.1, we may take $C(z \cdot C 1)=\beta \varphi_{b}$ where $\beta \in \mathbb{T}$ and $b \in \mathbb{D}$. Therefore we obtain

$$
C T_{z^{n}} C=T_{\left(\beta \varphi_{b}\right)^{n}}, \quad n=0,1,2, \cdots .
$$

It follows that $C T_{z^{n}} C\left(e_{b}\right)=T_{\left(\beta \varphi_{b}\right)^{n}}\left(e_{b}\right)$ and hence

$$
C\left(z^{n} \cdot C e_{b}\right)=\left(\beta \varphi_{b}\right)^{n} e_{b}
$$

for all $n=0,1,2, \cdots$. Note that $\left\{\left(\beta \varphi_{b}\right)^{n} e_{b}\right\}$ is an ONB of $H^{2}$. Then $\left\{z^{n} \cdot C e_{b}\right\}$ is also an ONB of $H^{2}$. Thus

$$
\int_{\mathbb{T}} z^{n} C e_{b} \overline{z^{m} C e_{b}} d \sigma=\int_{\mathbb{T}} z^{n-m}\left|C e_{b}\right|^{2} d \sigma=0
$$

for any nonnegative integers $n, m$ with $n \neq m$. Thus we get

$$
\int_{\mathbb{T}} z^{k}\left(\left|C e_{b}\right|^{2}-1\right) d \sigma=0
$$

for any integer $k$ because $\left\|C e_{b}\right\|=\left\|e_{b}\right\|=1$. This implies that $\left|C e_{b}\right|=$ 1 a.e. on $\mathbb{T}$ and then $C e_{b}$ is inner. Since $\left\{z^{n} C e_{b}\right\}$ is an ONB of $H^{2}$, we have

$$
H^{2}=\overline{\operatorname{span}}\left\{z^{n} C e_{b}: n=0,1,2, \cdots\right\}=C e_{b} \cdot H^{2},
$$

which implies that $C e_{b}=\alpha$, a unimodular constant. Thus (4.10) becomes

$$
C z^{n}=\alpha\left(\beta \varphi_{b}\right)^{n} e_{b}, \quad n=0,1,2, \cdots .
$$

Now Proposition 2.7 shows $C=\gamma C_{\lambda, a}$ for some $\lambda, \gamma \in \mathbb{T}$ and $a \in \mathbb{D}$. The proof is complete.

\section{ACKNOWLEDGEMENTS}

The first author was supported by NSFC (11771401) and the second author was supported by Basic Science Research Program through the National Research Foundation of Korea(NRF) funded by the Ministry of Education(NRF-2019R1I1A3A01041943). 


\section{REFERENCES}

[1] A. Brown, P. Halmos, Algebraic properties of Toeplitz operator, J. Reine Angew. Math. 213 (1964), 89-102.

[2] Q. Bu, Y. Chen, S. Zhu, Complex symmetric Toeplitz operators, Integr. Equ. Oper. Theory 93 (2021), Paper No. 15, 19pp.

[3] L. A. Coburn, Weyl's theorem for nonnormal operators, Michigan Math. J. 13 (1966), 285-288.

[4] C. C. Cowen, On equivalence of Toeplitz operators, J. Operator Theory 7 (1982), 167-172.

[5] R. G. Douglas, Banach Algebra Techniques in Operator Theory, Second edition, Springer, 1998.

[6] S. R. Garcia, J. E. Tener, Unitary equivalence of a matrix to its transpose, J. Operator Theory 68 (2012), 179-203.

[7] S. R. Garcia, W. R. Wogen, Complex symmetric partial isometries, J. Funct. Anal. 257 (2009), 1251-1260.

[8] K. Guo, Y. Ji, S. Zhu, A $C^{*}$-algebra approach to complex symmetric operators, Trans. Amer. Math. Soc. 367 (2015), 6903-6942.

[9] K. Guo, S. Zhu, A canonical decomposition of complex symmetric operators, J. Operator Theory 72 (2014), 529-547.

[10] P. R. Halmos, A Linear Algebra Problem Book, Dolciani Math. Exp., vol. 16, Math. Assoc. America, Washington, DC, 1995.

[11] Y. Gao, Z. Zhou, Complex symmetric composition operators induced by linear fractional maps, Indiana Univ. Math. J. 69 (2020), no. 2, 367-384.

[12] S. Jung, Y. Kim, E. Ko, J. E. Lee, Complex symmetric weighted composition operators on $H^{2}(\mathbb{D})$, J. Funct. Anal. 267 (2014), 323-351.

[13] E. Ko, J. E. Lee, On complex symmetric Toeplitz operators, J. Math. Anal. Appl. 434 (2016), 20-34.

[14] R. Li, Y. Yang, Y. Lu, A class of complex symmetric Toeplitz operators on Hardy and Bergman spaces, J. Math. Anal. Appl. 489 (2020), no. 2, 124173.

[15] R. Lim, L. H. Khoi, Complex symmetric weighted composition operators on $\mathcal{H}_{\gamma}(\mathbb{D})$, J. Math. Anal. Appl. 464 (2018), 101-118.

[16] S. K. Narayan, D. Sievewright, D. Thompson, Complex symmetric composition operators on $H^{2}$, J. Math. Anal. Appl. 443 (2016), 625-630.

[17] S. Noor, Complex symmetry of Toeplitz operators with continuous symbols, Arch. Math. 109 (2017), 455-460.

[18] M. Wang, K. Han, Complex symmetric weighted composition operators in several variables, J. Math. Anal. Appl. 474 (2019), 961-987.

[19] S. Zhu, Complex symmetric triangular operators, Oper. Matrices 9 (2015), $365-381$.

[20] S. Zhu, C. G. Li, Complex symmetric weighted shifts, Trans. Amer. Math. Soc. 365 (2013), 511-530. 
Department of Mathematics, Hangzhou Normal University, Hangzhou, 311121, P.R.CHINA

Email address: ychen@hznu.edu.cn

Department of Mathematics, Chonnam National University, Gwangue, 61186, KOREA

Email address: leeyj@chonnam.ac.kr

Department of Mathematics, Hangzhou Normal University, Hangzhou, 311121, P.R.CHINA

Email address: yilezhao@hznu.edu.cn 